Article

\title{
Environmentally-Friendly Extraction of Flavonoids from Cyclocarya paliurus (Batal.) Iljinskaja Leaves with Deep Eutectic Solvents and Evaluation of Their Antioxidant Activities
}

\author{
Xianchao Shang ${ }^{1,2}$, Jia-Neng Tan ${ }^{1, *}$, Yongmei Du ${ }^{1}$, Xinmin Liu ${ }^{1}$ and Zhongfeng Zhang ${ }^{1, *}$ \\ 1 Tobacco Research Institute of Chinese Academy of Agricultural Sciences, Qingdao 266101, China; \\ sxc3220342@163.com (X.S.); duyongmei@caas.cn (Y.D.); liuxinmin@caas.cn (X.L.) \\ 2 Graduate School of Chinese Academy of Agricultural Sciences, Beijing 100081, China \\ * Correspondence: tanjianeng@caas.cn (J.-N.T.); zhangzhongfeng@caas.cn (Z.Z.); Tel.: +86-532-88702052 \\ (J.-N.T.); Fax: +86-532-88702239 (J.-N.T.)
}

Received: 26 July 2018; Accepted: 14 August 2018; Published: 22 August 2018

\begin{abstract}
Deep eutectic solvents (DESs) are commonly employed as environmentally-friendly solvents in numerous chemical applications owing to their unique physicochemical properties. In this study, a novel and environmentally-friendly extraction method based on ultrasound assisted-deep eutectic solvent extraction (UAE-DES) was investigated for the extraction of flavonoids from Cyclocarya paliurus (Batal.) Iljinskaja (C. paliurus) leaves, and the antioxidant activities of these flavonoids were evaluated. Nine different DES systems based on either two or three components were tested, and the choline chloride/1,4-butanediol system (1:5 molar ratio) was selected as the optimal system for maximizing the flavonoid extraction yields. Other extraction conditions required to achieve the maximum flavonoid extraction yields from the leaves of $C$. paliurus were as follows: DES water content $(v / v), 30 \%$; extraction time, $30 \mathrm{~min}$; temperature, $60{ }^{\circ} \mathrm{C}$; and solid-liquid ratio, $20 \mathrm{mg} / \mathrm{mL}$. Liquid chromatography-mass spectrometry allowed the detection of five flavonoids in the extract, namely kaempferol-7-O- $\alpha$-L-rhamnoside, kaempferol, quercetin, quercetin-3-O- $\beta$-D-glucuronide, and kaempferol-3-O- $\beta$-D-glucuronide. In vitro antioxidant tests revealed that the flavonoid-containing extract exhibited strong DPPH and ABTS radical-scavenging abilities. Results indicate that UAE-DES is a suitable approach for the selective extraction of flavonoids from C. paliurus leaves, and DESs can be employed as sustainable extraction media for other bioactive compounds.
\end{abstract}

Keywords: Cyclocarya paliurus; deep eutectic solvent; extraction; flavonoid; antioxidant activity

\section{Introduction}

Cyclocarya paliurus (Batal.) Iljinskaja (C. paliurus), which belongs to the genus Cyclocarya Iljinskaja (Juglangdaceae), is a unique and state-protected plant species [1,2]. This plant is widely distributed in the mountains, valleys, and limestone mountains at around 420 to $2500 \mathrm{~m}$ above sea level in southern China [3]. Interestingly, the leaves of $C$. paliurus traditionally have been used as a health food [4-6], which may be related to its high content of biologically active compounds. Indeed, previous studies have revealed that the leaves of $C$. paliurus contain flavonoids, polysaccharides, internal esters, sterols, saponins, amino acids, and various mineral elements [7-10]. More specifically, flavonoids have been recognized as the main active compounds in C. paliurus, and these compounds exhibit a range of biological activities, which have attracted significant attention over the recent years $[7,8,11]$. To date, five main flavonoids have been isolated from C. paliurus leaves, 
including kaempferol-7-O- $\alpha$-L-rhamnoside, kaempferol, quercetin, quercetin-3-O- $\beta$-D-glucuronide, and kaempferol-3-O- $\beta$-D-glucuronide [12].

Conventional organic solvent-based techniques for the extraction of flavonoids tend to require long extraction times and high extraction temperatures, with the large-scale use of organic solvents being detrimental to the environment. To address these issues, deep eutectic solvents (DESs) have been applied in several areas of chemistry [13-18], including various extraction and purification processes. Deep eutectic solvents are environmentally-friendly solvents that exhibit a variety of beneficial properties. For example, they are simple to prepare from cheap starting materials, and their remarkable physicochemical and biodegradable properties render them versatile alternatives to conventional organic solvents [19-21]. Besides being biodegradable and non-toxic, DESs reduce the use of petroleum solvent, they have technical performances comparable with those of volatile organic solvents. Moreover, DESs are very suitable to microwave or ultrasound-assisted extraction, because this polar solvents have a higher dielectric constant than organic solvents and can absorb more energy, which would result in better extraction yields and saving energy [22]. Using DESs to extract phenolic compounds from oil can avoid the production of waste water containing phenolic compounds and the use of inorganic acids and alkalis [14]. Deep eutectic solvents consist of simple and naturally occurring compounds with a high safety profile, and they are considered as new environmentally-friendly solvents.

As such, DESs have been employed in the extraction and separation of various bioactive substances such as alkaloids, phenolic compounds, terpenoids, and saponins from plant materials [23-30]. Dai et al. [20] studied the extraction of flavonols from safflower, using different DESs: glucose: choline chloride; lactic acid: glucose and fructose: glucose: sucrose. Hu et al. [31] evaluated the efficiency of 43 DESs in the green extraction of different types of natural compounds from five Chinese herbal medicines. The results indicated that most DESs proved to be efficient solvents for extraction of alkaloids. Duarte et al. [32] present their results of the extraction of phenolic compounds from green coffee beans using different DESs solutions. The results were compared to extractions made using acetone and citric acid solution, it was found that DESs have a high ability to extract phenolic compounds from green coffee beans, which is related with the H-bond interactions that are established between the phenolic compounds and the DESs molecules. All these studies demonstrated that DESs can be used as suitable extraction solvents for selectively and efficiently extracting bioactive compounds from various plant sources.

Conventional techniques to obtain flavonoids, such as heating, boiling, or refluxing, usually require long extraction times and high extraction temperatures, which result in low extraction efficiency due to hydrolysis, ionization, and oxidation during extraction process [33]. Many new extraction technologies have been developed to separate flavonoids from plants, such as ultrasound-assisted extraction (UAE) [25,31], microwave-assisted extraction (MAE) [29], and supercritical extraction [34]. Supercritical extraction (including subcritical water extraction, supercritical fluid extraction, pressurized fluid extraction, and accelerated solvent extraction) was proved to be a suitable technique for isolation of flavonoids [35,36]. Ultrasound-assisted extraction and MAE are more known to be a fast and efficient method for the extraction of flavonoids from plants. Recently, the MAE system was successfully applied for extraction of flavonoids form C. paliurus [37]. Herein, we investigate the efficiency of a wide range of DES systems of different compositions for the extraction of flavonoids from C. paliurus leaves using the UAE system. To the best of our knowledge, rapid DES-based UAE-assisted extraction of this class of flavonoids has yet to be reported. Optimization of the extraction conditions was also carried out, with a focus on variables such as the water content in the DES, the extraction time and temperature, and the solid-liquid ratio, with the overall aim to maximize the flavonoid yield. In addition, the antioxidant activities of the various flavonoids are determined using the DPPH (1,1-dipheny-2-picrylhydrazyl) and ABTS (2,2'-azinobis-(3-ethylbenzthiazoline-6-sulphonate)) radical-scavenging assays. Overall, our main objective is to establish an efficient and environmentally friendly DES-based method for the extraction of flavonoids from C. paliurus leaves. 


\section{Experimental}

\subsection{Chemicals and Reagents}

Kaempferol (K, purity > 99\%), quercetin (Q, purity > 99\%), kaempferol-7-O- $\alpha$-L-rhamnoside (KRha, purity $>99 \%$ ), quercetin-3-O- $\beta$-D-glucuronide (QGlu, purity $>99 \%$ ), and kaempferol-3-O- $\beta$-D-glucuronide (KGlu, purity $>99 \%$ ) standards were obtained from Shanghai Yuan Mu Biotechnology Co., Ltd. (Shanghai, China). Choline chloride, lactic acid, glucose, glycerol, 1,4-butanediol, citric acid, malic acid, urea, xylitol, and malonic acid were purchased from Aladdin Reagent Company (Shanghai, China). 1,1-Dipheny-2-picrylhydrazyl (DPPH) and 2,2'-azinobis-(3-ethylbenzthiazoline-6-sulphonate) (ABTS) were obtained from Shanghai Hao Ocean Biological Technology Co., Ltd. (Shanghai, China). HPLC-grade acetonitrile and methanol were purchased from Shanghai Macklin Biochemical Co., Ltd. (Shanghai, China). Butylated hydroxytoluene (BHT, purity $>98 \%$ ) and vitamin C (VC, purity $>98 \%$ ) were obtained from Qingdao Si Yuan Chemical Co., Ltd. (Qingdao, China).

\subsection{Plant Materials}

The leaves of $C$. paliurus were obtained from Suining County, Jiangxi Province, China. The leaf specimen $(100 \mathrm{~g})$ was sliced into small pieces, frozen at $-80^{\circ} \mathrm{C}$, and freeze-dried over $48 \mathrm{~h}$ using a freeze-dryer (Biosafer, Shanghai, China). The freeze-dried leaf portions were ground into a fine powder (60 mesh) using a mill, and stored at $4{ }^{\circ} \mathrm{C}$ prior to use.

\subsection{Preparation of the DESs}

The desired DESs were prepared by mixing two or three components at a specific molar ratio under magnetic agitation for $1-4 \mathrm{~h}$ at $60-80^{\circ} \mathrm{C}$ until a clear and homogeneous liquid was obtained. Nine different DES systems were prepared, as outlined in Table 1.

Table 1. Employed DES systems.

\begin{tabular}{ccccc}
\hline \multirow{2}{*}{ DES Code } & \multicolumn{3}{c}{ Component } & \multirow{2}{*}{ Molar Ratio } \\
\cline { 2 - 4 } & $\mathbf{1}$ & $\mathbf{2}$ & $\mathbf{3}$ & \\
\hline DES-1 & choline chloride & glucose & - & $2: 1$ \\
DES-2 & choline chloride & citric acid & - & $1: 1$ \\
DES-3 & choline chloride & glycerol & - & $1: 1$ \\
DES-4 & choline chloride & urea & - & $1: 1$ \\
DES-5 & choline chloride & citric acid & glycerol & $1: 1: 1$ \\
DES-6 & choline chloride & 1,4-butanediol & - & $1: 5$ \\
DES-7 & choline chloride & lactic acid & - & $1: 1$ \\
DES-8 & choline chloride & malonic acid & - & $1: 1$ \\
DES-9 & choline chloride & malic acid & xylosic alcohol & $1: 1: 1$ \\
\hline
\end{tabular}

\subsection{Optimization of the Extraction Procedure}

Different DESs were tested under the same condition using the same protocol (solid to solvent ratio, $20 \mathrm{mg} / \mathrm{mL}$; extraction time, $20 \mathrm{~min}$; extraction temperature, $\left.50^{\circ} \mathrm{C}\right)$. A $70 \%$ DES solution in water $(v / v)$ was employed in the initial screening, precisely weighted $40 \mathrm{mg}$ of leaf sample powder was added to $2 \mathrm{~mL}$ of the extraction solvent in a $20 \mathrm{~mL}$ test tube. The resulting mixture was subjected to ultrasonication $(200 \mathrm{~W})$ at $50{ }^{\circ} \mathrm{C}$ for $20 \mathrm{~min}$ prior to centrifugation at $9200 \times \mathrm{g}$ for $10 \mathrm{~min}$. The obtained suspension was made up to a volume of $5 \mathrm{~mL}$ using either acetonitrile or methanol prior to UHPLC-MS analysis. Each extraction was performed in triplicate, and the extraction yield $\left(E_{y}\right)$ was calculated as follows:

$$
E_{y}=C_{f} \times V_{s} / m_{s}
$$

where $C_{f}$ is the concentration of each compound present in the DES, $V_{S}$ is the dilute suspension volume, and $m_{S}$ is the mass of the sample. 
Optimization of the extraction procedure was carried out using the following parameters: type of DES: as indicated in Table 1; DES water content: 10, 20, 30, 40, or 50\%; extraction time: 10, 20, 30, 40, or 50 min; extraction temperature: $30,40,50,60$, or $70^{\circ} \mathrm{C}$; and solid (sample powder)-liquid (extractant) ratio: $10,20,40,60$, or $80 \mathrm{mg} / \mathrm{mL}$.

\subsection{Liquid Chromatography-Mass Spectrometry (LC-MS) Conditions}

Following their extraction from the leaves of C. paliurus, the various flavonoids were analyzed by liquid chromatography-mass spectrometry (LC-MS). The mobile phase consisted of methanol (eluent A) and water containing $0.1 \%$ formic acid (eluent B). The gradient program was as follows: 0-1 min, 10\% B; 1-12.5 min, 10-35\% B; 12.5-13 min, 35-45\% B; 13-14 min, 45-35\% B; 14-15 min, 35-10\% B. The flow rate was $0.3 \mathrm{~mL} / \mathrm{min}$, the injection volume was $5 \mu \mathrm{L}$ and the column temperature was maintained at $25^{\circ} \mathrm{C}$. Owing to the high viscosity of the DESs, the eluent was discharged to the waste for the first $3.5 \mathrm{~min}$ of each run to protect the ion source.

Liquid chromatography-mass spectrometry was performed in the multiple-reaction monitoring (MRM) and positive electrospray ionization (ESI) modes. The gas temperature was set at $350{ }^{\circ} \mathrm{C}$, and a nitrogen flow rate of $8 \mathrm{~L} / \mathrm{min}$ was employed along with a nebulizer pressure of 35 psi. All other ESI and MS parameters were optimized individually for each target compound.

\subsection{Recovery of Extracted Bioactive Compounds from DES Extracts}

Brominated-type macroporous resins (SP-270) were tested to recover bioactive compounds from DES extracts. The glass column $(25 \mathrm{~mm} \times 600 \mathrm{~mm})$ was wet-packed with $5 \mathrm{~g}$ of SP-270 resins. Following extraction under the optimal conditions, the sample solution $(2 \mathrm{~mL})$ was loaded onto the column. After washing with deionized water $(20 \mathrm{~mL})$, the sample solution was eluted with ethanol $(30 \mathrm{~mL})$ at a constant flow rate of $10 \mathrm{~mL} / \mathrm{min}$. All ethanol fractions were dried prior to application in the antioxidant activity tests.

\subsection{DPPH Radical-Scavenging Activity}

The radical-scavenging activity of the flavonoid-containing extract was initially examined using the DPPH assay according to the method reported by Pinteus et al. [38,39]. Ten different sample concentrations (i.e., $10-100 \mu \mathrm{g} / \mathrm{mL})$ were examined. The desired sample $(0.1 \mathrm{~mL})$ was added to a solution of DPPH $(0.1 \mathrm{~mL}, 0.2 \mathrm{mM})$ dissolved in $95 \%$ ethanol, then shaken and allowed to stand at $25{ }^{\circ} \mathrm{C}$ for $30 \mathrm{~min}$ in the absence of light. The absorbance of each sample was measured at $517 \mathrm{~nm}$ using a Sunrise absorbance microplate reader (Tecan, Boston, MA, USA), and the radical-scavenging activities were compared to those of known antioxidants, including butylated hydroxytoluene (BHT) and vitamin C (VC). All samples were measured in triplicate. The percentage reduction of the DPPH radical content was expressed as per the following equation:

$$
\text { DPPH radical-scavenging activity }(\%)=\left[\left(A_{\text {blank }}-A_{\text {sample }}\right) / A_{\text {blank }}\right] \times 100 \%
$$

where $A_{\text {blank }}$ is the absorbance of the control (i.e., a mixture of all reagents minus the test compound), and $A_{\text {sample }}$ is the absorbance of the test mixture.

Based on the percentage reduction of the DPPH radical content, $\mathrm{IC}_{50}$, an index for comparison of the antioxidant activities, was calculated using SPSS 17.0 software.

\subsection{ABTS Radical-Scavenging Activity}

The radical-scavenging activity of the flavonoid-containing extract was determined using the ABTS assay according to the procedure described by Sun et al. [40,41]. The ABTS radical cation employed herein was prepared by mixing equal volumes of 7-mM ABTS solution and 2.45-mM potassium persulfate solution. The resulting solution was incubated for $12-14 \mathrm{~h}$ at $25^{\circ} \mathrm{C}$ in the absence of light, and subsequently diluted with ethanol to give an absorbance of $0.70 \pm 0.05$ units at $734 \mathrm{~nm}$. Ten different sample 
concentrations were examined here (i.e., $10-100 \mu \mathrm{g} / \mathrm{mL})$. Thus, the desired sample $(0.05 \mathrm{~mL})$ was added to the ABTS solution $(0.15 \mathrm{~mL})$ over $30 \mathrm{~min}$ in the absence of light and allowed to stand until the absorbance became stable. The absorbance was measured at $734 \mathrm{~nm}$ using a Sunrise absorbance microplate reader (Tecan, Boston, MA, USA). Each measurement was carried out in triplicate. Vitamin C and BHT were used as positive controls. The percentage reduction of the ABTS radical content was estimated as follows:

$$
\text { ABTS radical-scavenging activity }(\%)=\left[\left(A_{\text {blank }}-A_{\text {sample }}\right) / A_{\text {blank }}\right] \times 100 \%
$$

where $A_{\text {blank }}$ is the absorbance of the control (i.e., a mixture of all reagents minus the test compound) and $A_{\text {sample }}$ is the absorbance of the test mixture.

Based on the percentage reduction in the ABTS content, the $\mathrm{IC}_{50}$ value was determined, as described above.

\subsection{Statistical Analysis}

All experiments were performed in triplicate. For data analysis, the SPSS 17.0 software package (SPSS, Chicago, IL, USA) was used to detect statistically significant differences between means [7,11]. Differences associated with $p$ values lower than 0.05 were considered significant.

\section{Results and Discussion}

\subsection{LC-MS Analysis of the Flavonoids}

Mass spectrometric analysis of the flavonoids was initially performed over the full scan under both positive and negative ion modes. However, negative mode ESI was selected as the optimal condition since it produced higher responses. In addition, the $[\mathrm{M}-\mathrm{H}]^{-}$ion was chosen as the precursor ion for all analytes because of its high relative intensity, and the fragmentor was optimized. Subsequently, the product ions of the various compounds were selected and the collision energy for each ion transition was optimized (Table 2). The fragmentation patterns of the various flavonoids were compared with those reported in the literature previously (Supplemental Figure S1) [4,7,10].

Liquid chromatography-mass spectrometry was employed to determine the concentrations of the extracted flavonoids, and the abbreviations, retention times $\left(t_{R}\right)$, molecular weights (MW), mass-to-charge $(\mathrm{m} / \mathrm{z})$ values $(\mathrm{MS})$, and $\mathrm{MS}^{2}$ fragmentation ions of the flavonoids are listed in Table 2. The five constituent flavonoids were identified as kaempferol (K), kaempferol-7-O- $\alpha$-L-rhamnoside (KRha), quercetin (Q), quercetin-3-O- $\beta$-D-glucuronide (QGlu), and kaempferol-3-O- $\beta$-D-glucuronide (KGlu). The calibration curve equations, linearity ranges, limits of detection (LODs), limits of quantitation (LOQs), and coefficients of determination of the standard solutions are listed in Table 3.

Table 2. Retention times, MWs, $m / z$ values, and $\mathrm{MS}^{2}$ fragmentation ions of the five flavonoids detected in C. paliurus leaves.

\begin{tabular}{ccccc}
\hline Flavonoid & $\mathbf{t}_{\mathbf{R}}(\mathbf{m i n})$ & MW & $\boldsymbol{m} / \boldsymbol{z}$ Value & MS $^{2}$ Fragment Ions $(\boldsymbol{m} / \boldsymbol{z})$ \\
\hline QGlu & 4.83 & 478.37 & 477.10 & $150.99,178.95,300.94$ \\
KGlu & 5.80 & 462.36 & 461.19 & $133.14,199.00,285.05$ \\
KRha & 6.89 & 432.09 & 431.17 & $227.02,255.02,283.89$ \\
Q & 8.39 & 302.23 & 301.00 & $107.00,121.01,151.02$ \\
K & 10.26 & 286.23 & 285.01 & $117.76,186.99,239.00$ \\
\hline
\end{tabular}

Table 3. Calibration curve equations, linearity ranges, LODs, and LOQs of the standard solutions.

\begin{tabular}{cccccc}
\hline Flavonoid & Regression Equation & Linear Range $(\mu \mathrm{g} / \mathrm{g})$ & Coefficient of Determination $\left(\mathbf{r}^{2}\right)$ & LOD $(\mu \mathrm{g} / \mathrm{g})$ & $\mathbf{L O Q}(\boldsymbol{\mu g} / \mathrm{g})$ \\
\hline K & $\mathrm{y}=33570 \mathrm{x}+5715$ & $0.01-0.5$ & 0.9997 & 0.002 \\
Q & $\mathrm{y}=37130 \mathrm{x}+2490$ & $0.01-0.5$ & 0.9994 & 0.007 \\
KRha & $\mathrm{y}=12705 \mathrm{x}+3487$ & $0.5-25$ & 0.9999 & 0.008 \\
KGlu & $\mathrm{y}=27780 \mathrm{x}+32736$ & $0.1-5$ & 0.9995 & 0.008 \\
QGlu & $\mathrm{y}=49660 \mathrm{x}+23127$ & $0.5-25$ & 0.9992 & 0.012 \\
\hline
\end{tabular}




\subsection{Optimization of the DES Extraction Parameters}

\subsubsection{Influence of the DES Composition}

In order to determine the effect of the DES composition on the extraction of the five flavonoids from C. paliurus leaves, nine different choline chloride-based DES systems were examined, which contained glucose, citric acid, glycerol, urea, citric acid, glycerol,1,4-butanediol, lactic acid, malonic acid, malic acid, or xylosic alcohol as hydrogen bond donor. The viscosity of the extraction solvent was reduced by the addition of water, with a 70\% aqueous solution of DES being employed for the initial screening. The conditions employed for the extraction were as follows: solid-liquid ratio, $20 \mathrm{mg} / \mathrm{mL}$; extraction time, $20 \mathrm{~min}$; extraction temperature, $50^{\circ} \mathrm{C}$. As outlined in Table 4, significant differences in the extractabilities of the various flavonoids were observed by LC-MS for the various DES compositions employed herein.

Table 4. Influence of the DES composition on the extraction yield.

\begin{tabular}{cccccc}
\hline \multirow{2}{*}{ DES System } & \multicolumn{5}{c}{ Extraction Yields of the Target Compounds (mg/g) } \\
\cline { 2 - 6 } & $\mathbf{Q}$ & K & KRha & KGlu & QGlu \\
\hline DES-1 & $0.007 \pm 0.001^{\mathrm{d}}$ & $0.018 \pm 0.001^{\mathrm{g}}$ & $1.59 \pm 0.02^{\mathrm{f}}$ & $0.23 \pm 0.01^{\mathrm{d}}$ & $2.60 \pm 0.02^{\mathrm{d}}$ \\
DES-2 & $0.021 \pm 0.001^{\mathrm{c}}$ & $0.044 \pm 0.002^{\mathrm{d}}$ & $2.74 \pm 0.02^{\mathrm{b}}$ & $0.25 \pm 0.01^{\mathrm{bc}}$ & $2.25 \pm 0.01^{\mathrm{e}}$ \\
DES-3 & $0.011 \pm 0.000^{\mathrm{d}}$ & $0.027 \pm 0.001^{\mathrm{f}}$ & $2.14 \pm 0.01^{\mathrm{e}}$ & $0.21 \pm 0.00^{\mathrm{d}}$ & $2.85 \pm 0.02^{\mathrm{c}}$ \\
DES-4 & $0.010 \pm 0.001^{\mathrm{d}}$ & $0.021 \pm 0.002^{\mathrm{fg}}$ & $2.73 \pm 0.01^{\mathrm{b}}$ & $0.23 \pm 0.03^{\mathrm{c}}$ & $3.01 \pm 0.05^{\mathrm{b}}$ \\
DES-5 & $0.025 \pm 0.001^{\mathrm{bc}}$ & $0.036 \pm 0.001^{\mathrm{e}}$ & $2.46 \pm 0.01^{\mathrm{d}}$ & $0.25 \pm 0.00^{\mathrm{b}}$ & $2.84 \pm 0.02^{\mathrm{c}}$ \\
DES-6 & $0.033 \pm 0.002^{\mathrm{a}}$ & $0.105 \pm 0.004^{\mathrm{a}}$ & $3.11 \pm 0.05^{\mathrm{a}}$ & $0.32 \pm 0.02^{\mathrm{a}}$ & $3.59 \pm 0.04^{\mathrm{a}}$ \\
DES-7 & $0.024 \pm 0.001^{\mathrm{bc}}$ & $0.045 \pm 0.002^{\mathrm{d}}$ & $2.62 \pm 0.03^{\mathrm{c}}$ & $0.13 \pm 0.01^{\mathrm{f}}$ & $2.14 \pm 0.03^{\text {ef }}$ \\
DES-8 & $0.027 \pm 0.001^{\mathrm{b}}$ & $0.067 \pm 0.000^{\mathrm{c}}$ & $2.76 \pm 0.01^{\mathrm{b}}$ & $0.24 \pm 0.01^{\mathrm{cd}}$ & $2.12 \pm 0.04^{\mathrm{f}}$ \\
DES-9 & $0.025 \pm 0.000^{\mathrm{bc}}$ & $0.094 \pm 0.007^{\mathrm{b}}$ & $2.66 \pm 0.02^{\mathrm{c}}$ & $0.20 \pm 0.01^{\mathrm{e}}$ & $2.62 \pm 0.01^{\mathrm{d}}$ \\
\hline
\end{tabular}

Data are expressed in the form of mean \pm standard deviation $(n=3)$. Values in the same column that do not share the same letter are significantly different (one way ANOVA and Tukey's test, $p<0.05$ ). Extraction conditions: water content, $30 \%$; extraction time, $20 \mathrm{~min}$; extraction temperature, $50^{\circ} \mathrm{C}$; solid-liquid ratio, $20 \mathrm{mg} / \mathrm{mL}$; ultrasonic power, $200 \mathrm{~W}$.

Clearly, the extraction capacity of the tested DESs varied greatly depending on the type of HBD in DES. Amine- and alcohol-based DESs were superior to those of the sugar- and carboxylic acid-based DESs. In addition, as flavonoids are readily soluble in alkaline solvents, the extraction efficiencies of organic acid-based DESs were lower than those of amine- or alcohol-based DESs. In general, extraction yields of the five flavonoids followed the order QGlu $>$ KRha $>K G l u>K>Q$. The flavonoid quercetin-3-O- $\beta$-D-glucuronide was highly extractable in DES-6 (3.59 mg/g), followed by DES-3 (2.85 mg/g), and DES-4 (3.01 mg/g), whereas DES-2 (2.25 mg/g), DES-7 (2.14 mg/g), DES-8 $(2.12 \mathrm{mg} / \mathrm{g})$, and DES-9 $(2.62 \mathrm{mg} / \mathrm{g})$ demonstrated lower extractability for QGlu. For flavonoid kaempferol-7-O- $\alpha$-L-rhamnoside, DES-6 (3.11 mg/g) led to higher extraction yields, followed by (DES-2, DES-4, DES-7, DES-8, DES-9), lower extraction yields were obtained in sugar-based DES-1 (1.59 mg/g). For flavonoid kaempferol-3-O- $\beta$-D-glucuronide, DES-6 (0.32 mg/g) showed higher extraction efficiency, while the other DESs shared the same extraction yields except DES-7 (0.13 mg/g). For flavonoids kaempferol and quercetin, DES-2, DES-7, DES-8, DES-9 exhibited higher extraction efficiency than DES-4 and DES-6. The highest quantities of flavonoids were extracted using the solvent combinations of choline chloride:urea (1:1 molar ratio) and choline chloride:1,4-butanediol (1:5 molar ratio). Thus, as a result of the lower cost of 1,4-butanediol, the choline chloride:1,4-butanediol system was selected as the optimal extraction solvent.

We also found that the flavonoid extraction yields decreased upon reducing the choline chloride:1,4-butanediol ratio. Specifically, when this ratio was decreased further to 1:6, the extraction yields decreased (Supplemental Figure S2). In terms of the mechanism of extraction, it has been reported that the alcohol-based hydrogen bond donor and the flavonoid molecules compete for the chloride anion. These species can envelope the chloride anion, resulting in considerable steric hindrance and preventing interactions between the target compounds and the chloride anion. Further decrease in the choline chloride content in the DES would therefore result in insufficient chloride anions 
being present to interact with the target compounds. However, a low 1,4-butanediol content would result in issues relating to both diffusion and mass transfer, and so DES-6, comprised of choline chloride and 1,4-butanediol in a 1:5 molar ratio, was selected as the optimal solvent for subsequent experiments.

\subsubsection{Effect of the DES Water Content}

Experiments were conducted to evaluate the effect of the water content in the DES on the flavonoid yields. Thus, DES-6 (choline chloride:1,4-butanediol, 1:5 molar ratio) containing different water contents (i.e., 10, 20, 30, 40, or $50 \mathrm{vol} \%$ ) was employed for the extraction, which was carried out over $20 \mathrm{~min}$ at $50{ }^{\circ} \mathrm{C}$ using a solid-liquid ratio of $20 \mathrm{mg} / \mathrm{mL}$.

The influence of different DES water contents can be seen clearly in Figures. In Figure 1A,B, where it is apparent that the extraction yield depended strongly on the water content. Clearly, upon increasing the water content from 10 to $30 \%$, significant increases in the extraction efficiencies were observed for all five flavonoids. This observation can be most likely attributed to the lower viscosity of the DES, which reduces intermolecular interactions within the solvent and gives rise to increased molecular motion and solubility. However, a further increase in the water content led to decreased extraction efficiencies, and thus water content of 30\% was considered as optimal and was employed for all subsequent experiments (extraction yields: $\mathrm{Q}=0.034 \mathrm{mg} / \mathrm{g}, \mathrm{K}=0.117 \mathrm{mg} / \mathrm{g}, \mathrm{KRha}=3.183 \mathrm{mg} / \mathrm{g}$, $\mathrm{KGlu}=0.331 \mathrm{mg} / \mathrm{g}$, and QGlu $=3.628 \mathrm{mg} / \mathrm{g}$ ). We believe that high water contents may be interfering with the interactions between the flavonoids and the DES systems.
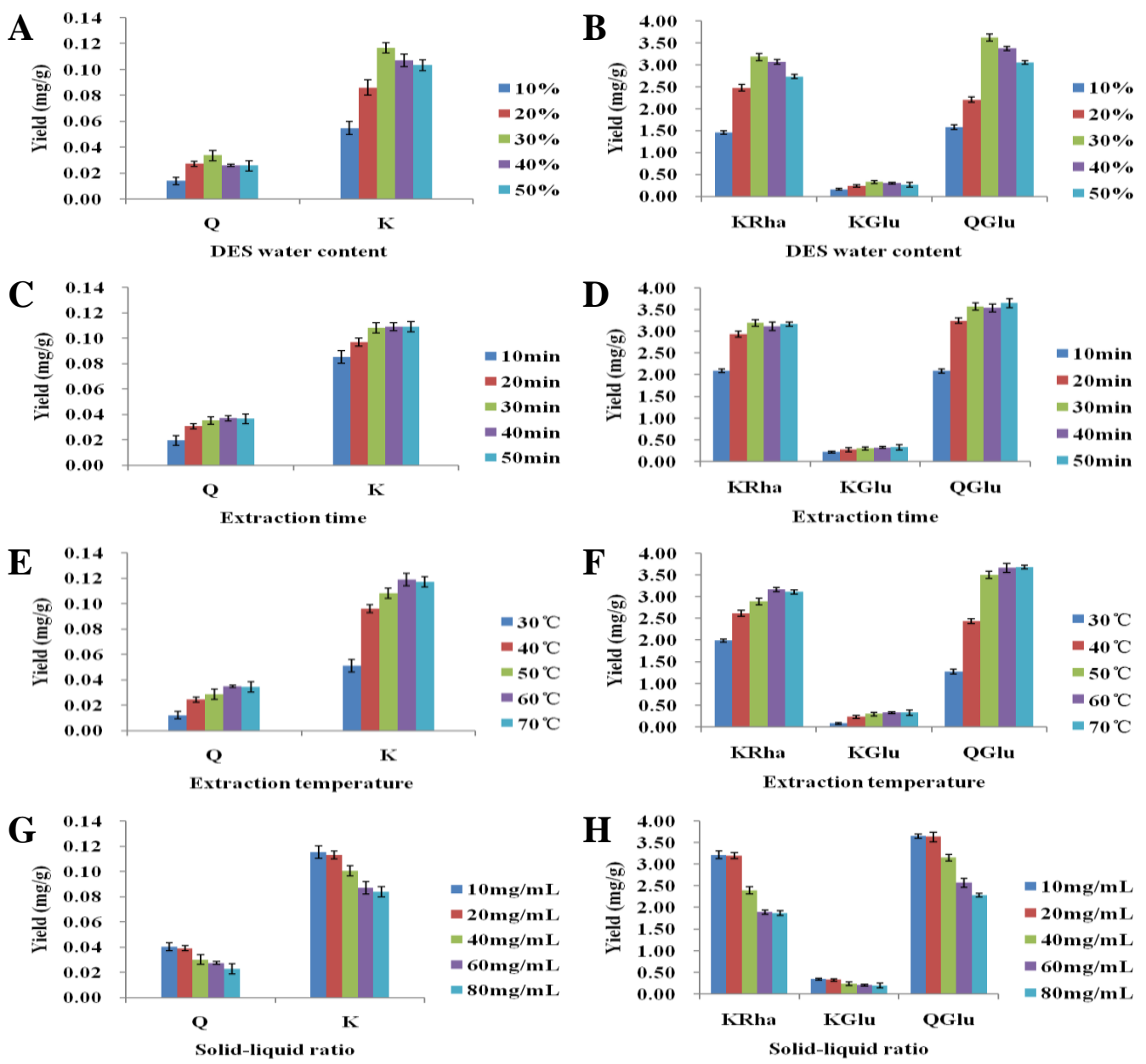

Figure 1. The effect of the water content in the DES, extraction time, temperature, and the solid-liquid ratio on the extraction yield: $(\mathbf{A}, \mathbf{B})$ water content in the DES, $(\mathbf{C}, \mathbf{D})$ extraction time, $(\mathbf{E}, \mathbf{F})$ extraction temperature, and $(\mathbf{G}, \mathbf{H})$ solid-liquid ratio. 


\subsubsection{Effect of the Extraction Time}

To determine the effect of the extraction time on the obtained flavonoid yields, the samples were extracted over 10, 20,30, 40, or $50 \mathrm{~min}$, while all other conditions were maintained constant (i.e., DES water content, $30 \%$; extraction temperature, $50{ }^{\circ} \mathrm{C}$; solid-liquid ratio, $20 \mathrm{mg} / \mathrm{mL}$ ).

The effect of the extraction time on the obtained flavonoid yields is shown in Figure 1C,D. As expected, short extraction times (i.e., 10 or $20 \mathrm{~min}$ ) resulted in low extraction efficiencies, likely as a result of the limited flavonoid dissolution in the extraction solvent. However, upon increasing the extraction time to $30 \mathrm{~min}$, significant improvements in the extraction efficiencies were observed. Further increasing the extraction time had no remarkable effect, as dissolution was essentially complete after $30 \mathrm{~min}$. Based on these results, an optimal extraction time of $30 \mathrm{~min}$ was employed for all subsequent experiments.

\subsubsection{Effect of the Extraction Temperature}

To determine the optimum extraction temperature for flavonoid extraction, leaf samples were extracted using $30 \mathrm{vol} \%$ DES- 6 over $20 \mathrm{~min}$ at $30,40,50,60$, or $70^{\circ} \mathrm{C}$, and the results are outlined in Figure 1E,F. As indicated, upon increasing the extraction temperature from 30 to $60{ }^{\circ} \mathrm{C}$, a clear increase in the flavonoid yields was observed, although no further increases took place at higher temperatures (i.e., $70^{\circ} \mathrm{C}$ ). An extraction temperature of $60^{\circ} \mathrm{C}$ was therefore employed for further experiments.

\subsubsection{Effect of the Solid-Liquid Ratio}

A range of solid (sample mass)-liquid (extractant volume) ratios were also examined (i.e., $10-80 \mathrm{mg} / \mathrm{mL}$ ) to determine the optimal conditions for extraction. Interestingly, we found that the flavonoid recoveries tended to increase upon decreasing the ratio from 80 to $20 \mathrm{mg} / \mathrm{mL}$, as shown in Figure 1G,H. This phenomenon was attributed to the excellent extraction capacity of the DES system. When a large sample mass was employed, the extraction capacity of the DES solvent was low, making the extraction slow. By contrast, when a lower sample mass was used, the extraction capacity of the solvent was increased, and a shorter extraction time could be employed. However, at lower solid-liquid ratios (i.e., $10-20 \mathrm{mg} / \mathrm{mL}$ ), no further increase in the extraction yield was observed. This result can be most likely attributed to complete dissolution of the flavonoids. We therefore determined that a solid-liquid ratio of $20 \mathrm{mg} / \mathrm{mL}$ was optimal.

In addition, second-order kinetics for extraction of flavonoids from C. paliurus with deep eutectic solvents were also investigated. The results exhibited good linear relationship $\left(r^{2}>0.98\right)$ between the time/yield and time, indicating satisfactory extraction behaviors of deep eutectic solvents from plant system (Supplemental Table S1 and Figure S3).

\subsection{Evaluation of the Antioxidant Activity}

\subsubsection{DPPH Radical-Scavenging Activity}

To evaluate the antioxidant (or radical-scavenging) activity of the flavonoids extracted from the C. paliurus leaves, we analyzed their DPPH radical-scavenging activity. For a visual contrast, VC and BHT were used as positive controls and the five flavonoids determined were used as comparison, the results are shown in Figure 2A. Indeed, it was clear that the extracted flavonoids exhibited a clear DPPH radical-scavenging activity, with a positive correlation between the radical-scavenging activity and the flavonoid concentration. More specifically, the antioxidant activity of the extract increased from $29.32 \pm 1.23 \%$ to $96.61 \pm 1.01 \%$ upon increasing the concentration from 10 to $100 \mu \mathrm{g} / \mathrm{mL}$. In addition, a higher antioxidant activity was observed for the extracted flavonoids than for BHT at all concentrations, with an $\mathrm{IC}_{50}$ value of $25.2 \mu \mathrm{g} / \mathrm{mL}$. However, a superior radical-scavenging activity was observed for $\mathrm{VC}$, a typical antioxidant, which gave an $\mathrm{IC}_{50}$ value of $7.1 \mu \mathrm{g} / \mathrm{mL}$. With the increase of concentration, especially when the concentration reach $80 \mu \mathrm{g} / \mathrm{mL}$, the extracts share a similar DPPH radical-scavenging activity with the five flavonoids determined. Statistical analysis results indicate that 
DPPH radical-scavenging activity of extracts has no difference with quercetin $(\mathrm{Q})$. Based on the above results, it can be concluded that the flavonoids extracted from C. paliurus leaves using our optimized DES system exhibit considerable DPPH radical-scavenging activity.
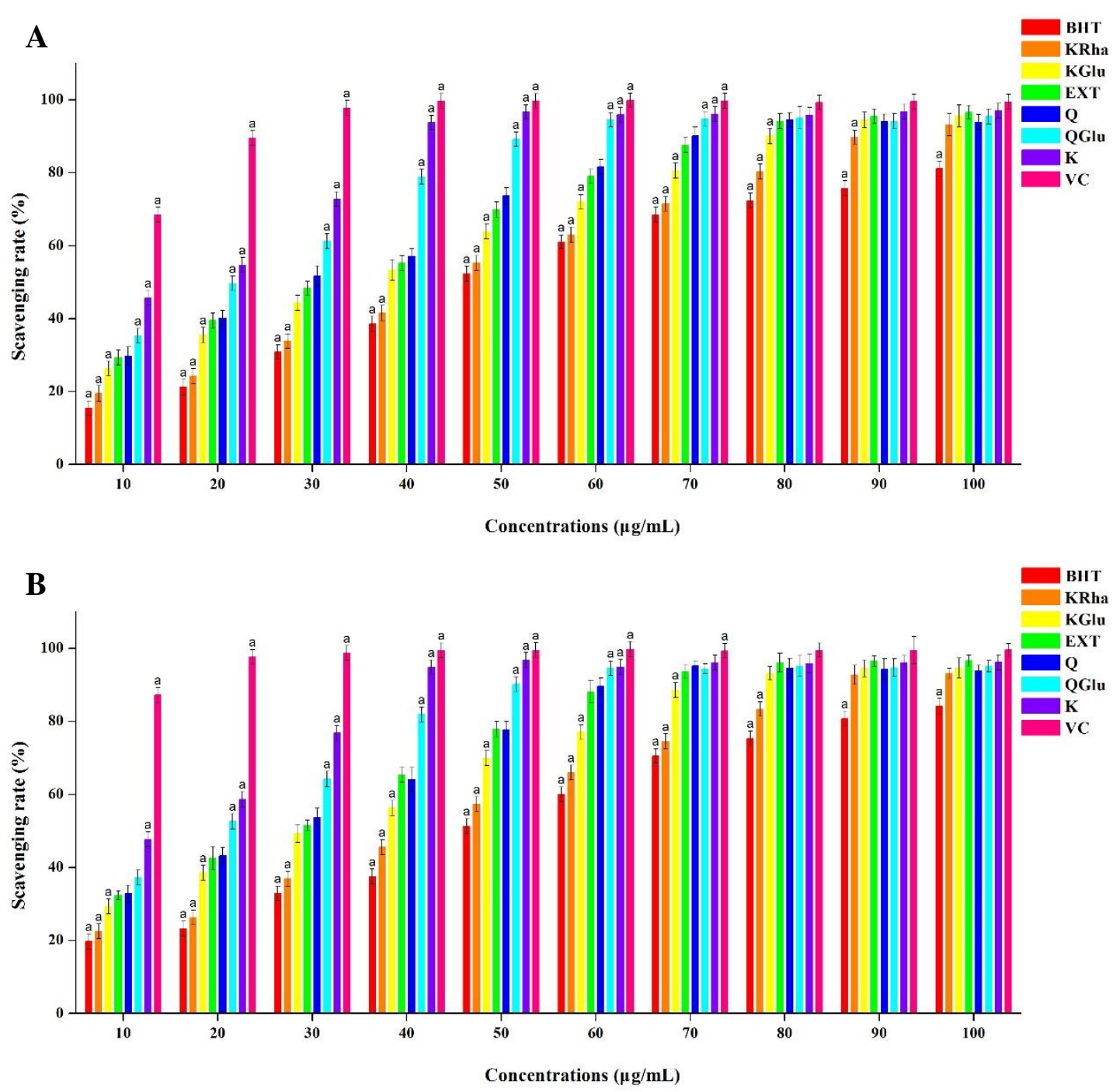

Figure 2. Antioxidant activities of the flavonoids extracted from C. paliurus leaves: (A) the DPPH radical-scavenging capacity and (B) the ABTS radical-scavenging capacity.BHT, butylated hydroxytoluene; KRha, kaempferol-7-O- $\alpha$-L-rhamnoside; KGlu, kaempferol-3-O- $\beta$-D-glucuronide; Ext, extraction from C. paliurus; $Q$, quercetin; $Q G l u$, quercetin-3-O- $\beta$-D-glucuronide; $K$, Kaempferol; VC, vitamin C. Scavenging rates that were significantly different from EXT are indicated with a $(p<0.05)$.

\subsubsection{ABTS Radical-Scavenging Activity}

We next examined the ABTS radical-scavenging activity of the flavonoid extracts over a range of concentrations. As indicated in Figure 2B, the radical-scavenging activity was enhanced at higher flavonoid concentrations, and a significantly higher activity was obtained than for BHT over the concentration range examined. However, as in the case of the DPPH assay, VC exhibited a superior scavenging activity towards the ABTS radical at concentrations between 10 and $100 \mu \mathrm{g} / \mathrm{mL}$. Also, ABTS radical-scavenging activity of extracts has no difference with quercetin $(\mathrm{Q})$ at concentrations between 10 and $100 \mu \mathrm{g} / \mathrm{mL}$. These results confirmed that the flavonoids extracted from C. paliurus leaves using our DES system exhibit an excellent antioxidant activity, with an $\mathrm{IC}_{50}$ value of $22.4 \mu \mathrm{g} / \mathrm{mL}$. We can conclude from the results of the DPPH and ABTS assays that our DES system is suitable for 
the extraction of flavonoids from C. paliurus leaves, and that the obtained extracts exhibit excellent antioxidant activities. As such, C. paliurus leaves can be considered as a valuable natural source of antioxidants in the human diet, in addition to their potential for use as functional foods or medicines.

\section{Conclusions}

A novel, simple, and environmentally-friendly UAE-DES method was developed for the extraction of five flavonoids from the leaves of C. paliurus. We found that choline chloride:1,4-butanediol in a 1:5 molar ratio was the optimal DES system, and this composition was considered to be advantageous because of its facile preparation, in addition to its relatively non-toxic and environmentally-friendly properties. Optimization of the extraction conditions maximized the extraction yields of the five flavonoids (i.e., kaempferol-7-O- $\alpha$-L-rhamnoside, kaempferol, quercetin, quercetin-3-O- $\beta$-D-glucuronide, and kaempferol-3-O- $\beta$-D-glucuronide), which were identified by LC-MS. The final optimized conditions were as follows: DES system, choline chloride:1,4-butanediol (1:5 molar ratio); DES water content, 30\%; extraction time, $30 \mathrm{~min}$; extraction temperature, $60^{\circ} \mathrm{C}$; and solid-liquid ratio, $20 \mathrm{mg} / \mathrm{mL}$. Under the optimal extraction conditions, the flavonoids obtained from the C. paliurus leaves exhibited good in vitro antioxidant activities in both DPPH and ABTS radical-scavenging assays $\left(\mathrm{IC}_{50}=25.2 \mu \mathrm{g} / \mathrm{mL}\right.$ for the DPPH assay, and $22.4 \mu \mathrm{g} / \mathrm{mL}$ for the ABTS assay). We therefore conclude that the developed DES system is a suitable and environmentally-friendly alternative for the extraction of flavonoids and other bioactive compounds from a range of plant species. Further studies are currently under way to elucidate the interactions between the flavonoids compounds and the various DESs.

Supplementary Materials: The following are available online, Figure S1: The UPLC-MS/MS TIC spectrum of the standards (A) and target compounds (B): kaempferol $(\mathrm{K}, 10.26 \mathrm{~min})$, quercetin $(\mathrm{Q}, 8.39)$, kaempferol-7$O$ - $\alpha$-L-rhamnoside (KRha, $6.89 \mathrm{~min})$, kaempferol-3-O- $\beta$-D-glucuronide (KGlu, 5.80), quercetin-3-O- $\beta$-D-glucuronide (QGlu, $4.83 \mathrm{~min}$ ); Figure S2: The effects of different ratios of choline chloride/1,4-butanediol on the yields of five flavonoids; Figure S3: Linear fittings of five flavonoids; Table S1: Linear fitting equations of five flavonoids.

Author Contributions: J.-N.T. and Z.Z. conceived and designed the experiments; X.S. and J.-N.T. performed the experiments; X.S., J.-N.T., Y.D. and Z.Z. analyzed the data; Y.D. and X.L. collected materials; J.-N.T. and Z.Z. wrote the paper and organized the whole research of this study. All authors approved the final version manuscript.

Funding: This work was supported by the Agricultural Science and Technology Innovation Program (ASTIP-TRIC05), the Science Foundation for Young Scholars of TRI-CAAS (2016B03), the Shandong Provincial Natural Science Foundation, China (ZR2016BQ43), and the Opening Project of the Key Laboratory of Oilseeds processing, Ministry of Agriculture (201704).

Conflicts of Interest: The authors declare no conflict of interest.

\begin{tabular}{|c|c|}
\hline ABTS & 2,2'-azinobis-(3-ethylbenzthiazoline-6-sulphonate) \\
\hline ANOVA & analysis of variance \\
\hline BHT & butylated hydroxytoluene \\
\hline DES & deep eutectic solvent \\
\hline DPPH & 1,1-dipheny-2-picrylhydrazyl \\
\hline ESI & electrospray ionization \\
\hline K & kaempferol \\
\hline KGlu & kaempferol-3-O- $\beta$-D-glucuronide \\
\hline KRha & kaempferol-7-O- $\alpha$-L-rhamnoside \\
\hline $\mathrm{IC}_{50}$ & concentration of antioxidant where the response is reduced by half \\
\hline MRM & multiple-reaction monitoring \\
\hline QGlu & quercetin-3-O- $\beta$-D-glucuronide \\
\hline SD & standard deviation \\
\hline$t_{R}$ & retention time \\
\hline UAE & ultrasound-assisted extraction \\
\hline UAE-DES & ultrasound assisted-deep eutectic solvent extraction \\
\hline $\mathrm{VC}$ & vitamin C \\
\hline
\end{tabular}




\section{References}

1. Xie, J.H.; Shen, M.Y.; Xie, M.Y.; Nie, S.P.; Chen, Y.; Li, C.; Huang, D.F.; Wang, Y.X. Ultrasonic-assisted extraction, antimicrobial and antioxidant activities of Cyclocarya paliurus (Batal.) Iljinskaja polysaccharides. Carbohyd. Polym. 2012, 89, 177-184. [CrossRef] [PubMed]

2. Wang, Z.J.; Xie, J.H.; Shen, M.Y.; Tang, W.; Wang, H.; Nie, S.P.; Xie, M.Y. Carboxymethylation of polysaccharide from Cyclocarya paliurus and their characterization and antioxidant properties evaluation. Carbohyd. Polym. 2016, 136, 988-994. [CrossRef] [PubMed]

3. Xie, M.Y.; Xie, J.H. Review about the research on Cyclocarya paliurus (Batal.) Iljinskaja. J. Food Sci. Bio. Technol. 2008, 27, 113-121.

4. Xie, M.Y.; Yu, Y.L.; Wang, Y.X.; Yi, X.; Wang, X.R. A study on the structure and contents of flavonoids in the leaves of Cyclocarya paliurus (Batal.) Iljinsk. Chin. J. Anal. Chem. 2003, 17, 1053-1056.

5. Xie, J.H.; Shen, M.Y.; Nie, S.P.; Liu, X.; Yin, J.Y.; Huang, D.F.; Zhang, H.; Xie, M.Y. Simultaneous analysis of 18 mineral elements in Cyclocarya paliurus polysaccharide by ICP-AES. Carbohyd. Polym. 2013, 94, 216-220. [CrossRef] [PubMed]

6. Xie, J.H.; Xie, M.Y.; Nie, S.P.; Shen, M.Y.; Wang, Y.X.; Li, C. Isolation, chemical composition and antioxidant activities of a water-soluble polysaccharide from Cyclocarya paliurus (Batal.) Iljinskaja. Food Chem. 2010, 119, 1626-1632. [CrossRef]

7. Jiang, C.H.; Yao, N.; Wang, Q.Q.; Zhang, J.H.; Sun, Y.; Xiao, N.; Liu, K.; Huang, F.; Fang, S.Z.; Shang, X.L.; et al. Cyclocarya paliurus extract modulates adipokine expression and improves insulin sensitivity by inhibition of inflammation in mice. J. Ethnopharmacol. 2014, 153, 344-351. [CrossRef] [PubMed]

8. Kurihara, H.; Asami, S.; Shibata, H.; Fukami, H.; Tanaka, T. Hypolipemic effect of Cyclocarya paliurus (Batal) Iljinskaja in lipid-loaded mice. Biol. Pharm. Bull. 2003, 26, 383-385. [CrossRef] [PubMed]

9. Deng, B.; Shang, X.L.; Fang, S.Z.; Li, Q.Q.; Fu, X.X.; Su, J. Integrated effects of light intensity and fertilization on growth and flavonoid accumulation in Cyclocarya paliurus. J. Agric. Food Chem. 2012, 60, 6286-6292. [CrossRef] [PubMed]

10. Cao, Y.N.; Fang, S.Z.; Yin, Z.Q.; Fu, X.X.; Shang, X.L.; Yang, W.X.; Yang, H.M. Chemical fingerprint and multicomponent quantitative analysis for the quality evaluation of Cyclocarya paliurus Leaves by HPLC-Q-TOF-MS. Molecules 2017, 22, 1927. [CrossRef] [PubMed]

11. Xie, J.H.; Wang, W.J.; Dong, C.J.; Huang, L.X.; Wang, H.; Li, C.; Nie, S.P.; Xie, M.Y. Protective effect of flavonoids from Cyclocarya paliurus leaves against carbon tetrachloride-induced acute liver injury in mice. Food Chem. Toxicol. 2018. [CrossRef] [PubMed]

12. Li, L.; Xie, M.Y.; Wang, X.R.; Lee, F.S.C. Determination of speciation of elements related to blood sugar in bioactive extracts from Cyclocarya paliurus leaves by FIA-ICP-MS. Eur. Food Res. Technol. 2006, 223, 202-209.

13. Abbott, A.P.; Cullis, P.M.; Gibson, M.J.; Harris, R.C.; Raven, E. Extraction of glycerol from biodiesel into a eutectic based ionic liquid. Green Chem. 2007, 9, 868-872. [CrossRef]

14. Hou, Y.C.; Kong, J.; Ren, Y.H.; Ren, S.H.; Wu, W.Z. Mass transfer dynamics in the separation of phenol from model oil with quaternary ammonium salts via forming deep eutectic solvents. Sep. Purif. Technol. 2017, 174, 554-560. [CrossRef]

15. Dai, Y.T.; Spronsen, J.V.; Witkamp, G.J.; Verpoorte, R.; Choi, Y.H. Natural deep eutectic solvents as new potential media for green technology. Anal. Chim. Acta 2013, 766, 61-68. [CrossRef] [PubMed]

16. Oliveira, F.S.; Pereiro, A.B.; Rebelo, L.P.N.; Marrucho, I.M. Deep eutectic solvents as extraction media for azeotropic mixtures. Green Chem. 2013, 15, 1326-1330. [CrossRef]

17. Bi, W.T.; Tian, M.L.; Row, K.H. Evaluation of alcohol-based deep eutectic solvent in extraction and determination of flavonoids with response surface methodology optimization. J. Chromatogr. A 2013, 1285, 22-30. [CrossRef] [PubMed]

18. Nam, M.W.; Zhao, J.; Lee, M.S.; Jeong, J.H.; Lee, J.M. Enhanced extraction of bioactive natural products using tailor-made deep eutectic solvents: Application to flavonoid extraction from Flos sophorae. Green Chem. 2014, 17, 1718-1727. [CrossRef]

19. Liu, Y.; Friesen, J.B.; McAlpine, J.B.; Lankin, D.C.; Chen, S.N.; Pauli, G.F. Natural Deep Eutectic Solvents: Properties, Applications, and Perspectives. J. Nat. Prod. 2018, 81, 679-690. [CrossRef] [PubMed] 
20. Dai, Y.T.; Verpoorte, R.; Choi, Y.H. Natural deep eutectic solvents providing enhanced stability of natural colorants from safflower (Carthamus tinctorius). Food Chem. 2014, 159, 116-121. [CrossRef] [PubMed]

21. Morrison, H.G.; Sun, C.C.; Neervannan, S. Characterization of thermal behavior of deep eutectic solvents and their potential as drug solubilization vehicles. Int. J. Pharmaceut. 2009, 378, 136-139. [CrossRef] [PubMed]

22. Khoddami, A.; Wilkes, M.A.; Roberts, T.H. Techniques for analysis of plant phenolic compounds. Molecules 2013, 18, 2328-2375. [CrossRef] [PubMed]

23. Ruesgas-Ramón, M.; Figueroa-Espinoza, M.C.; Durand, E. Application of Deep Eutectic Solvents (DES) for phenolic compounds extraction: Overview, challenges, and opportunities. J. Agric. Food Chem. 2017, 65, 3591-3601. [CrossRef] [PubMed]

24. Vieira, V.; Prieto, M.A.; Barros, L.; Coutinho, J.A.P.; Ferreira, I.C.F.R.; Ferreira, O. Enhanced extraction of phenolic compounds using choline chloride based deep eutectic solvents from Juglans regia L. Ind. Crop. Prod. 2018, 115, 261-271. [CrossRef]

25. Bajkacz, S.; Adamek, J. Evaluation of new natural deep eutectic solvents for the extraction of isoflavones from soy products. Talanta 2017, 168, 329-335. [CrossRef] [PubMed]

26. García, A.; Rodríguez-Juan, E.; Rodríguez-Gutiérrez, G.; Rios, J.J.; Fernández-Bolaños, J. Extraction of phenolic compounds from virgin olive oil by deep eutectic solvents (DESs). Food Chem. 2016, 197, 554-561. [CrossRef] [PubMed]

27. Peng, X.; Duan, M.H.; Yao, X.H.; Zhang, Y.H.; Zhao, C.J.; Zu, Y.G.; Fu, Y.J. Green extraction of five target phenolic acids from Lonicerae japonicae Flos with deep eutectic solvent. Sep. Purif. Technol. 2016, 157, $249-257$. [CrossRef]

28. Tang, B.K.; Bi, W.T.; Zhang, H.; Row, K.H. Deep eutectic solvent-based HS-SME coupled with GC for the analysis of bioactive terpenoids in Chamaecyparis obtuse leaves. Chromatographia 2014, 77, 373-377. [CrossRef]

29. Cui, Q.; Peng, X.; Yao, X.H.; Wei, Z.F.; Luo, M.; Wang, W.; Zhao, C.J.; Fu, Y.J.; Zu, Y.G. Deep eutectic solvent-based microwave-assisted extraction of genistin, genistein and apigenin from pigeon pea roots. Sep. Purif. Technol. 2015, 150, 63-72. [CrossRef]

30. Mukhopadhyay, S.; Mukherjee, S.; Hayyan, A.; Hayyan, M.; Hashim, M.A.; Gupta, B.S. Enhanced removal of lead from contaminated soil by polyol-based deep eutectic solvents and saponin. J. Contam. Hydrol. 2016, 194, 17-23. [CrossRef] [PubMed]

31. Duan, L.; Dou, L.L.; Guo, L.; Li, P.; Liu, E. Comprehensive evaluation of deep eutectic solvents in extraction of bioactive natural products. ACS Sustain. Chem. Eng. 2016, 4, 2405-2411. [CrossRef]

32. Paiva, A.; Craveiro, R.; Aroso, I.; Martins, M.; Rui, L.R.; Duarte, A.R.C. Natural deep eutectic solvents-solvents for the 21st century. ACS Sustain. Chem. Eng. 2014, 2, 1063-1071. [CrossRef]

33. Li, H.; Chen, B.; Yao, S.Z. Application of ultrasonic technique for extracting chlorogenic acid from Eucommia ulmodies Oliv. (E. ulmodies). Ultrason. Sonochem. 2005, 12, 295-300. [CrossRef] [PubMed]

34. Mojzer, E.B.; Hrnčič, M.K.; Škerget, M.; Knez, Ž.; Bren, U. Polyphenols: Extraction methods, antioxidative action, bioavailability and anticarcinogenic effects. Molecules 2016, 21, 901. [CrossRef] [PubMed]

35. Cai, Z.; Qu, Z.; Lan, Y.; Zhao, S.; Ma, X.; Wan, Q.; Jing, P.; Li, P. Conventional, ultrasound-assisted, and accelerated-solvent extractions of anthocyanins from purple sweet potatoes. Food Chem. 2016, 197, 266-272. [CrossRef] [PubMed]

36. Koyu, H.; Kazan, A.; Demir, S.; Haznedaroglu, M.Z.; Yesil-Celiktas, O. Optimization of microwave assisted extraction of Morus nigra L. fruits maximizing tyrosinase inhibitory activity with isolation of bioactive constituents. Food Chem. 2018, 248, 183-191. [CrossRef] [PubMed]

37. Xie, J.H.; Dong, C.J.; Nie, S.P.; Li, F.; Wang, Z.J.; Shen, M.Y.; Xie, M.Y. Extraction, chemical composition and antioxidant activity of flavonoids from Cyclocarya paliurus (Batal.) Iljinskaja leaves. Food Chem. 2015, 186, 97-105. [CrossRef] [PubMed]

38. Pinteus, S.; Silva, J.; Alves, C.; Horta, A.; Fino, N.; Rodrigues, A.I.; Mendes, S.; Pedrosa, R. Cytoprotective effect of seaweeds with high antioxidant activity from the Peniche coast (Portugal). Food Chem. 2017, 218, 591-599. [CrossRef] [PubMed]

39. Wang, Y.Q.; Gao, Y.J.; Ding, H.; Liu, S.J.; Han, X.; Gui, J.Z.; Liu, D. Subcritical ethanol extraction of flavonoids from Moringa oleifera leaf and evaluation of antioxidant activity. Food Chem. 2017, 218, 152-158. [CrossRef] [PubMed] 
40. Sun, T.; Powers, J.R.; Tang, J.M. Evaluation of the antioxidant activity of asparagus, broccoli and their juices. Food Chem. 2007, 105, 101-106. [CrossRef]

41. Vallverdú-Queralt, A.; Medina-Remón, A.; Andres-Lacueva, C.; Lamuela-Raventos, R.M. Changes in phenolic profile and antioxidant activity during production of diced tomatoes. Food Chem. 2011, 126, 1700-1707. [CrossRef] [PubMed]

Sample Availability: Samples of C. paliurus are available from the authors.

(C) 2018 by the authors. Licensee MDPI, Basel, Switzerland. This article is an open access article distributed under the terms and conditions of the Creative Commons Attribution (CC BY) license (http://creativecommons.org/licenses/by/4.0/). 